S6 Table. Genes detected as differentially expressed between slow and rapid progression PD patients identified by T-test

\title{
with permutations.
}

\begin{tabular}{|c|c|c|c|}
\hline Gene Symbol & $p$-value & T score & Degrees Freedom \\
\hline TOP1 & 61,2270 & 3,4396 & 0,0011 \\
\hline RNF165 & 59,0486 & 3,4409 & 0,0011 \\
\hline C11orf42 & 65,8680 & 3,2787 & 0,0017 \\
\hline RBKS & 65,0934 & $-3,1487$ & 0,0025 \\
\hline ASB18 & 50,2673 & 3,0450 & 0,0037 \\
\hline WDR72 & 60,9019 & $-2,9507$ & 0,0045 \\
\hline TPD52L1 & 65,9835 & $-2,9103$ & 0,0049 \\
\hline C1orf57 & 63,9309 & $-2,8794$ & 0,0054 \\
\hline FAM5C & 65,9968 & $-2,8606$ & 0,0057 \\
\hline PLA2G12B & 65,6354 & $-2,8338$ & 0,0061 \\
\hline RELL2 & 62,5825 & $-2,8341$ & 0,0062 \\
\hline ADIPOR2 & 55,5687 & $-2,8429$ & 0,0062 \\
\hline COX6A1 & 63,0467 & $-2,8233$ & 0,0064 \\
\hline HSP90AB6P & 64,8788 & $-2,7994$ & 0,0067 \\
\hline ZFP41 & 65,4827 & $-2,7972$ & 0,0068 \\
\hline RAD18 & 63,3168 & 2,7883 & 0,0070 \\
\hline ZNF284 & 65,8577 & 2,7831 & 0,0070 \\
\hline RSPH10B2 & 55,7316 & $-2,7819$ & 0,0074 \\
\hline OR2G3 & 63,6039 & $-2,7521$ & 0,0077 \\
\hline PCDHGB7 & 65,7603 & 2,7276 & 0,0082 \\
\hline LOC729815 & 64,2220 & $-2,7296$ & 0,0082 \\
\hline CBWD1 & 55,5128 & 2,7296 & 0,0085 \\
\hline ZNF221 & 62,4366 & 2,6818 & 0,0094 \\
\hline SPNS2 & 65,9605 & $-2,6741$ & 0,0094 \\
\hline HEATR1 & 53,8731 & $-2,6713$ & 0,0100 \\
\hline TRIM65 & 60,7050 & $-2,6570$ & 0,0101 \\
\hline PLAGL2 & 65,7821 & $-2,6501$ & 0,0101 \\
\hline RFESD & 53,9763 & $-2,6531$ & 0,0105 \\
\hline TBX2 & 59,4826 & 2,6316 & 0,0108 \\
\hline POLR2C & 63,0221 & 2,6254 & 0,0108 \\
\hline DCTN2 & 62,5472 & $-2,5969$ & 0,0117 \\
\hline C22orf34 & 64,0710 & $-2,5910$ & 0,0118 \\
\hline HIST1H2BJ & 53,8469 & 2,5910 & 0,0123 \\
\hline ZNF383 & 60,1479 & $-2,5720$ & 0,0126 \\
\hline FDX1L & 65,7994 & $-2,5360$ & 0,0136 \\
\hline MRM1 & 64,8558 & $-2,5245$ & 0,0140 \\
\hline ATP4A & 61,3776 & $-2,5247$ & 0,0142 \\
\hline ENKUR & 61,7512 & 2,5141 & 0,0146 \\
\hline
\end{tabular}

\begin{tabular}{|c|c|c|c|}
\hline Gene Symbol & p-value & T score & Degrees Freedom \\
\hline LHX5 & 62,6382 & 2,5128 & 0,0146 \\
\hline FTCD & 58,4678 & 2,5160 & 0,0146 \\
\hline OR2L13 & 57,2665 & 2,5074 & 0,0150 \\
\hline CCDC144C & 60,8114 & 2,4986 & 0,0152 \\
\hline MND1 & 54,0286 & 2,4916 & 0,0158 \\
\hline LOC100287301 & 65,4172 & $-2,4743$ & 0,0160 \\
\hline ANUBL1 & 65,5053 & $-2,4381$ & 0,0175 \\
\hline YY2 & 65,5499 & 2,4321 & 0,0178 \\
\hline ABCC11 & 55,5234 & $-2,4349$ & 0,0181 \\
\hline C16orf79 & 64,5141 & $-2,4244$ & 0,0181 \\
\hline HP1BP3 & 65,9537 & $-2,4227$ & 0,0182 \\
\hline TEKT1 & 51,0770 & 2,4357 & 0,0184 \\
\hline UBL4B & 65,9266 & $-2,4083$ & 0,0188 \\
\hline SMPD2 & 65,9223 & $-2,3989$ & 0,0193 \\
\hline CILP & 65,9932 & 2,3964 & 0,0194 \\
\hline BHLHE23 & 59,1935 & $-2,4026$ & 0,0194 \\
\hline CPT1A & 63,0919 & $-2,3886$ & 0,0199 \\
\hline SCG5 & 58,3503 & 2,3870 & 0,0202 \\
\hline TMEM132C & 60,0191 & 2,3781 & 0,0206 \\
\hline MED31 & 65,9999 & $-2,3720$ & 0,0206 \\
\hline ROM1 & 65,9886 & 2,3718 & 0,0206 \\
\hline MRPS15 & 62,6466 & $-2,3732$ & 0,0207 \\
\hline GTF2A1 & 64,8052 & $-2,3666$ & 0,0209 \\
\hline IGFALS & 65,5337 & $-2,3630$ & 0,0211 \\
\hline AVPR1B & 59,2307 & 2,3676 & 0,0212 \\
\hline SYCE2 & 51,7182 & 2,3689 & 0,0216 \\
\hline S100P & 47,6773 & $-2,3637$ & 0,0222 \\
\hline CC2D2B & 55,8382 & $-2,3511$ & 0,0223 \\
\hline PPAPDC3 & 58,8119 & $-2,3466$ & 0,0223 \\
\hline SCLY & 60,0739 & $-2,3351$ & 0,0229 \\
\hline HOMER2 & 56,5108 & 2,3355 & 0,0231 \\
\hline GRAPL & 60,4457 & $-2,3287$ & 0,0232 \\
\hline HIST2H4A & 63,1094 & 2,3208 & 0,0235 \\
\hline SENP2 & 64,9704 & 2,3087 & 0,0242 \\
\hline NMUR1 & 65,7426 & 2,3043 & 0,0244 \\
\hline SLC35D1 & 65,0559 & $-2,3034$ & 0,0245 \\
\hline WASH3P & 65,9794 & $-2,3018$ & 0,0245 \\
\hline USP31 & 54,3313 & $-2,3122$ & 0,0246 \\
\hline
\end{tabular}




\begin{tabular}{|c|c|c|c|}
\hline Gene Symbol & p-value & T score & Degrees Freedom \\
\hline C15orf48 & 65,7088 & $-2,3007$ & 0,0246 \\
\hline DOK7 & 65,6643 & $-2,2984$ & 0,0247 \\
\hline C1QTNF6 & 65,9243 & $-2,2969$ & 0,0248 \\
\hline TXNDC17 & 65,8547 & $-2,2942$ & 0,0250 \\
\hline OLFM2 & 62,2706 & $-2,2962$ & 0,0250 \\
\hline ZNF334 & 57,5153 & 2,2994 & 0,0251 \\
\hline $\mathrm{BCO} 2$ & 61,5984 & 2,2901 & 0,0255 \\
\hline FAM125A & 64,4071 & $-2,2841$ & 0,0257 \\
\hline GALK1 & 65,1379 & $-2,2802$ & 0,0259 \\
\hline DCBLD1 & 55,2065 & $-2,2867$ & 0,0261 \\
\hline HGSNAT & 63,3368 & 2,2775 & 0,0261 \\
\hline NMNAT3 & 65,9733 & $-2,2714$ & 0,0264 \\
\hline ELMO1 & 65,0687 & 2,2717 & 0,0264 \\
\hline RSPH6A & 65,7898 & $-2,2708$ & 0,0264 \\
\hline PVALB & 56,9925 & 2,2786 & 0,0265 \\
\hline SNRNP25 & 55,6348 & $-2,2758$ & 0,0267 \\
\hline GLB1L & 63,2591 & $-2,2664$ & 0,0269 \\
\hline C20orf152 & 65,5520 & 2,2617 & 0,0270 \\
\hline UBIAD1 & 64,2397 & $-2,2553$ & 0,0275 \\
\hline F8 & 60,3542 & 2,2535 & 0,0279 \\
\hline C1orf69 & 64,8596 & $-2,2480$ & 0,0280 \\
\hline HIST1H2BC & 65,9465 & 2,2458 & 0,0281 \\
\hline RDH16 & 57,8907 & $-2,2510$ & 0,0282 \\
\hline HMHB1 & 64,8833 & 2,2447 & 0,0282 \\
\hline ACTA2 & 63,6464 & 2,2442 & 0,0283 \\
\hline FAM194B & 62,7096 & 2,2445 & 0,0283 \\
\hline RSPH9 & 56,9343 & 2,2461 & 0,0286 \\
\hline OR5M1 & 59,2983 & $-2,2424$ & 0,0287 \\
\hline VMO1 & 60,3653 & $-2,2381$ & 0,0289 \\
\hline IPO13 & 65,5418 & $-2,2311$ & 0,0291 \\
\hline EPHX4 & 63,2092 & 2,2308 & 0,0292 \\
\hline LOC100131234 & 64,4239 & 2,2297 & 0,0293 \\
\hline RGS3 & 65,3163 & 2,2252 & 0,0295 \\
\hline ACTRT2 & 65,9187 & $-2,2113$ & 0,0305 \\
\hline OR10AD1 & 65,8074 & 2,2071 & 0,0308 \\
\hline RLBP1 & 56,9982 & $-2,2047$ & 0,0315 \\
\hline ZNF673 & 58,7474 & 2,2027 & 0,0316 \\
\hline MAS1L & 52,5722 & 2,2090 & 0,0316 \\
\hline MIPEP & 63,8787 & $-2,1900$ & 0,0322 \\
\hline CENPE & 65,8502 & $-2,1861$ & 0,0324 \\
\hline LCNL1 & 55,5553 & 2,1936 & 0,0325 \\
\hline ATAD3A & 60,7199 & $-2,1888$ & 0,0325 \\
\hline CDC42BPG & 63,4623 & $-2,1756$ & 0,0333 \\
\hline
\end{tabular}

\begin{tabular}{|c|c|c|c|}
\hline Gene Symbol & $\boldsymbol{p}$-value & T score & Degrees Freedom \\
\hline SIGLEC11 & 64,4072 & $-2,1698$ & 0,0337 \\
\hline CPA6 & 65,1281 & 2,1674 & 0,0339 \\
\hline HES4 & 65,6005 & $-2,1653$ & 0,0340 \\
\hline INSC & 51,0578 & 2,1770 & 0,0341 \\
\hline CHTF18 & 65,8707 & $-2,1567$ & 0,0347 \\
\hline PSTPIP2 & 55,8054 & 2,1632 & 0,0348 \\
\hline GUCA2A & 65,9959 & 2,1533 & 0,0350 \\
\hline RRN3 & 61,7803 & $-2,1490$ & 0,0356 \\
\hline CCR1 & 61,6880 & $-2,1480$ & 0,0356 \\
\hline CCDC7 & 49,9950 & 2,1591 & 0,0357 \\
\hline SLC16A14 & 61,8622 & 2,1447 & 0,0359 \\
\hline CD24 & 66,0000 & $-2,1388$ & 0,0362 \\
\hline AQP7 & 65,7881 & $-2,1375$ & 0,0363 \\
\hline KRT16P1 & 53,3805 & 2,1453 & 0,0365 \\
\hline RNASEH2C & 59,7589 & $-2,1386$ & 0,0366 \\
\hline TRIM31 & 65,8232 & $-2,1334$ & 0,0366 \\
\hline COL23A1 & 65,2066 & 2,1334 & 0,0367 \\
\hline NUDT7 & 60,2859 & 2,1326 & 0,0370 \\
\hline ZNF404 & 64,4336 & $-2,1271$ & 0,0372 \\
\hline C18orf56 & 62,5188 & $-2,1257$ & 0,0375 \\
\hline PFKM & 65,9165 & $-2,1233$ & 0,0375 \\
\hline ANKRD20A1 & 63,5924 & 2,1245 & 0,0375 \\
\hline ABCC1 & 62,1749 & $-2,1253$ & 0,0375 \\
\hline ABCC6 & 54,7024 & 2,1278 & 0,0379 \\
\hline KIAA1671 & 63,7586 & $-2,1189$ & 0,0380 \\
\hline COL2A1 & 53,3692 & $-2,1227$ & 0,0384 \\
\hline PIK3R5 & 64,0589 & 2,1138 & 0,0384 \\
\hline CALY & 63,8191 & 2,1135 & 0,0385 \\
\hline C20orf144 & 51,7667 & 2,1221 & 0,0386 \\
\hline CCBP2 & 64,0118 & $-2,1099$ & 0,0388 \\
\hline PTOV1 & 61,5686 & $-2,1097$ & 0,0390 \\
\hline OR1F1 & 56,0647 & 2,1141 & 0,0390 \\
\hline TTC7B & 65,8089 & 2,1061 & 0,0390 \\
\hline TRIM49 & 63,8621 & 2,1064 & 0,0391 \\
\hline ZNF256 & 64,9687 & 2,1051 & 0,0392 \\
\hline LOC100287497 & 61,6122 & $-2,1020$ & 0,0396 \\
\hline ST3GAL1 & 65,6427 & $-2,0989$ & 0,0397 \\
\hline DEFB116 & 63,4471 & 2,0998 & 0,0397 \\
\hline FSTM1 & 60,2405 & $-2,1003$ & 0,0399 \\
\hline GRHCI & 62,3506 & $-2,0983$ & 0,0399 \\
\hline 65,0633 & $-2,0954$ & 0,0400 \\
\hline HN3 & 59,9418 & 2,0970 & 0,0402 \\
\hline 57,1749 & 2,0981 & 0,0403 \\
\hline
\end{tabular}




\begin{tabular}{|c|c|c|c|}
\hline Gene Symbol & p-value & T score & Degrees Freedom \\
\hline LOC645954 & 65,3209 & 2,0790 & 0,0415 \\
\hline $\mathrm{PSPH}$ & 63,0480 & $-2,0805$ & 0,0416 \\
\hline C12orf44 & 55,0616 & $-2,0846$ & 0,0418 \\
\hline NDUFA8 & 64,6004 & $-2,0757$ & 0,0419 \\
\hline VPS35 & 61,7644 & $-2,0713$ & 0,0425 \\
\hline HEATR2 & 65,6147 & $-2,0659$ & 0,0428 \\
\hline C5orf45 & 65,3551 & $-2,0656$ & 0,0428 \\
\hline TCEAL3 & 65,7728 & $-2,0652$ & 0,0428 \\
\hline RPH3A & 63,3115 & 2,0656 & 0,0430 \\
\hline GPT2 & 64,4005 & $-2,0642$ & 0,0430 \\
\hline CROCC & 65,5650 & $-2,0632$ & 0,0431 \\
\hline CNTNAP1 & 60,6133 & $-2,0652$ & 0,0432 \\
\hline SCARF1 & 65,9228 & 2,0598 & 0,0434 \\
\hline PNMA1 & 65,9519 & 2,0568 & 0,0437 \\
\hline CALM3 & 65,2159 & 2,0569 & 0,0437 \\
\hline PDZD7 & 57,0182 & $-2,0617$ & 0,0438 \\
\hline CCDC99 & 63,0810 & $-2,0525$ & 0,0443 \\
\hline SNORA33 & 65,9988 & $-2,0504$ & 0,0443 \\
\hline WDR53 & 62,6156 & 2,0505 & 0,0445 \\
\hline DNMT3A & 65,9450 & $-2,0470$ & 0,0446 \\
\hline SHPK & 65,9515 & $-2,0470$ & 0,0447 \\
\hline OR8B4 & 54,1329 & 2,0524 & 0,0450 \\
\hline STAB1 & 58,6819 & $-2,0482$ & 0,0450 \\
\hline BMP2K & 65,9954 & $-2,0417$ & 0,0452 \\
\hline FAM155B & 65,4670 & $-2,0403$ & 0,0454 \\
\hline TSC2 & 65,9260 & $-2,0386$ & 0,0455 \\
\hline ATP11A & 64,4344 & 2,0382 & 0,0456 \\
\hline SSTR2 & 61,4255 & 2,0385 & 0,0458 \\
\hline AKR1CL1 & 65,8869 & $-2,0339$ & 0,0460 \\
\hline MFSD6L & 52,1824 & $-2,0417$ & 0,0463 \\
\hline
\end{tabular}

\begin{tabular}{|c|c|c|c|}
\hline Gene Symbol & $p$-value & T score & Degrees Freedom \\
\hline IFNA7 & 65,4780 & 2,0294 & 0,0465 \\
\hline DUOX1 & 48,2871 & 2,0409 & 0,0467 \\
\hline LOC100287314 & 62,3937 & 2,0271 & 0,0469 \\
\hline RESP18 & 55,3021 & 2,0303 & 0,0471 \\
\hline SYCP2L & 65,8209 & $-2,0217$ & 0,0473 \\
\hline CDK9 & 65,9558 & $-2,0193$ & 0,0475 \\
\hline OR6C74 & 63,6301 & 2,0201 & 0,0476 \\
\hline GSR & 65,9999 & 2,0152 & 0,0480 \\
\hline CCDC134 & 54,9172 & 2,0228 & 0,0480 \\
\hline PIWIL3 & 62,9610 & 2,0168 & 0,0480 \\
\hline HIST1H4H & 53,5535 & 2,0217 & 0,0482 \\
\hline INO80D & 65,9882 & 2,0127 & 0,0482 \\
\hline GOLGA8C & 65,9420 & 2,0115 & 0,0484 \\
\hline $\mathrm{HLA}-\mathrm{H}$ & 65,8970 & 2,0100 & 0,0485 \\
\hline VAMP2 & 65,8463 & $-2,0095$ & 0,0486 \\
\hline WISP2 & 65,9832 & $-2,0092$ & 0,0486 \\
\hline ZBTB12 & 60,2837 & $-2,0108$ & 0,0488 \\
\hline ABL2 & 65,9986 & $-2,0039$ & 0,0492 \\
\hline FLJ44881 & 59,4884 & 2,0072 & 0,0493 \\
\hline LOC100131642 & 65,9020 & 2,0027 & 0,0493 \\
\hline TMEM110 & 61,1851 & 2,0043 & 0,0495 \\
\hline LTF & 64,9529 & $-2,0019$ & 0,0495 \\
\hline MEIG1 & 52,8924 & 2,0105 & 0,0495 \\
\hline TXNDC2 & 53,8957 & $-2,0079$ & 0,0497 \\
\hline LEUTX & 61,6579 & $-2,0015$ & 0,0497 \\
\hline DEFB125 & 65,6011 & $-1,9990$ & 0,0498 \\
\hline RNF20 & 61,5760 & $-2,0013$ & 0,0498 \\
\hline LYPD6 & 56,4265 & 2,0038 & 0,0499 \\
\hline PCDHGB3 & 60,6312 & $-2,0005$ & 0,0499 \\
\hline
\end{tabular}

